\title{
(6) OPEN ACCESS \\ Haemodynamic effects of umbilical cord milking in premature sheep during the neonatal transition
}

\author{
Douglas A Blank, ${ }^{1,2}$ Graeme R Polglase, ${ }^{2}$ Martin Kluckow, ${ }^{3}$ Andrew William Gill, ${ }^{4}$ \\ Kelly J Crossley, ${ }^{2}$ Alison Moxham, ${ }^{2}$ Karyn Rodgers, ${ }^{2}$ Valerie Zahra, ${ }^{2}$ Ishmael Inocencio, ${ }^{2}$ \\ Fiona Stenning, ${ }^{2}$ Domeic A LaRosa, ${ }^{2}$ Peter G Davis, ${ }^{1}$ Stuart B Hooper ${ }^{2}$
}

\begin{abstract}
${ }^{1}$ Newborn Research, The Royal Women's Hospital, Parkville, Victoria, Australia

${ }^{2}$ The Ritchie Centre, Hudson Institute of Medical Research, Monash University, Clayton, Victoria, Australia

${ }^{3}$ Department of Neonatology, Royal North Shore Hospital and University of Sydney, New South Wales, Australia

${ }^{4}$ Centre for Neonatal Research and Education, University of Western Australia, Perth, Australia
\end{abstract}

Correspondence to Dr Douglas A Blank, The Ritchie Centre, Hudson Institute of Medical Research, Monash University, Clayton, Victoria 3168, Australia; blankdouglas@ gmail.com

Received 24 August 2017 Revised 31 October 2017 Accepted 5 November 2017 Published Online First

5 December 2017

\section{Linked}

- http://dx.doi.org/10.1136/ fetalneonatal-2017-313657

Check for updates

To cite: Blank DA, Polglase GR, Kluckow M, et al. Arch Dis Child Fetal Neonatal Ed 2018;103:F539-F546.

\section{ABSTRACT}

Objective Umbilical cord milking (UCM) at birth may benefit preterm infants, but the physiological effects of UCM are unknown. We compared the physiological effects of two UCM strategies with immediate umbilical cord clamping (UCC) and physiological-based cord clamping (PBCC) in preterm lambs.

Methods At 126 days' gestational age, fetal lambs were exteriorised, intubated and instrumented to measure umbilical, pulmonary and cerebral blood flows and arterial pressures. Lambs received either (1) UCM without placental refill (UCMwoPR); (2) UCM with placental refill (UCMwPR); (3) PBCC, whereby ventilation commenced prior to UCC; or (4) immediate UCC. UCM involved eight milks along a $10 \mathrm{~cm}$ length of cord, followed by UCC.

Results $A$ net volume of blood was transferred into the lamb during UCMwPR (8.8 mL/kg, IQR 8-10, P=0.01) but not during UCMwoPR ( $0 \mathrm{~mL} / \mathrm{kg}$, IQR -2.8 to 1.7$)$ or PBCC (1.1 mL/kg, IQR -1.3 to 4.3). UCM had no effect on pulmonary blood flow, but caused large fluctuations in mean carotid artery pressures (MBP) and blood flows (CABF). In UCMwoPR and UCMwPR lambs, MBP increased by $12 \% \pm 1 \%$ and $8 \% \pm 1 \%$ and $C A B F$ increased by $32 \% \pm 2 \%$ and $15 \% \pm 2 \%$, respectively, with each milk. Cerebral oxygenation decreased the least in PBCC lambs $(17 \%$, IQR $13-26)$ compared with UCMwoPR $(26 \%$, IQR $23-25, \mathrm{P}=0.03)$, UCMwPR (35\%, IQR 27-44, $\mathrm{P}=0.02)$ and immediate UCC $(34 \%$, IQR 28-41, $\mathrm{P}=0.02)$ lambs. Conclusions UCMwoPR failed to provide placental transfusion, and UCM strategies caused considerable haemodynamic disturbance. UCM does not provide the same physiological benefits of PBCC. Further review of UCM is warranted before adoption into routine clinical practice.

\section{INTRODUCTION}

Delayed umbilical cord clamping (UCC) improves outcomes for preterm infants. The benefits include fewer blood transfusions, lower rates of intraventricular haemorrhage and necrotising enterocolitis, improved haemodynamics, and improved motor function at $18-22$ months of age. ${ }^{1-4}$ However, if the newborn remains apnoeic after stimulation, international resuscitation guidelines recommend immediate UCC and moving the infant to a resuscitation platform to provide positive pressure ventilation (PPV)..$^{5-7}$ As over $60 \%$ of infants less than 29 weeks' gestation receive PPV in the delivery room, the opportunity for delayed UCC in these infants is

\section{What is already known on this topic?}

Umbilical cord milking has been investigated as an alternative method to delayed cord clamping in preterm infants.

- To date, no studies describe the physiological effects of umbilical cord milking at birth.

\section{What this study adds?}

- Umbilical cord milking in preterm lambs caused considerable haemodynamic disturbances in carotid artery blood flow and systemic blood pressure without increasing pulmonary blood flow.

- Neither umbilical cord milking without placental refill nor delayed cord clamping resulted in a placental transfusion, whereas umbilical cord milking with placental refill did.

- Umbilical cord milking does not provide the same physiological benefits of delayed cord clamping.

limited. ${ }^{89}$ It is widely assumed that the main benefit of delayed UCC is placental to infant blood transfusion, which has led to the suggestion that umbilical cord milking (UCM) may be an alternative to delayed UCC.

In humans, investigators have milked $20 \mathrm{~cm}$ of the umbilical cord (UC) over $2 \mathrm{~s}$, which is repeated two to four times. ${ }^{10-17}$ Proponents suggest that the primary advantage of UCM is the rapid transfer of blood from the placenta to the infant, which then does not delay the infant's transfer to a warming bed for respiratory support. However, this assumes that placental transfusion is the primary benefit of delayed UCC. Nevertheless, several trials have now concluded that UCM appears to confer the same benefits of delayed UCC ${ }^{111} 1^{18-20}$ and may be more effective in infants delivered by caesarean section. ${ }^{1321}$

It is widely recognised that lung aeration is the key to maintaining physiological homeostasis during neonatal resuscitation as it is a requirement for pulmonary gas exchange and stimulates an increase in pulmonary blood flow (PBF). ${ }^{5-7} 22-27$ Recent animal studies have demonstrated that ventilation prior to UCC ('physiological-based cord clamping' or PBCC) has benefits independent of a 
placental transfusion, including maintaining cardiac output and stabilising arterial blood pressures during transition. ${ }^{28} 29$ PBCC involves aerating the lung, stimulating a large increase in PBF, so that pulmonary venous return can immediately replace umbilical venous return as the primary source of left ventricular preload following UCC. PBCC also mitigates the large increase in arterial pressure and cerebral blood flow that results from the increase in afterload associated with UCC. ${ }^{28}{ }^{29}$ As a result, lambs receiving PBCC have more stable mean systemic blood pressures (MBP), mean carotid artery blood flows (CABF), cerebral oxygenation $\left(\mathrm{SctO}_{2} \%\right)$ and heart rate $(\mathrm{HR})$ compared with lambs receiving immediate UCC.

No information is currently available on the physiological effects of UCM and whether it is physiologically similar to delayed UCC. We used preterm lambs to study the physiological changes induced by two UCM strategies. These were UCM without placental refill (UCMwoPR), which is most similar to UCM performed in human infants, ${ }^{10-16}$ and UCM with placental refill (UCMwPR) to ensure placental blood transfusion prior to UCC. To our knowledge, no clinical trial has specifically used a strategy of UCMwPR in humans. These UCM techniques were compared with PBCC and immediate UCC to investigate how each influenced PBF, MBP, CABF, SctO \%, HR and UC blood flow. We hypothesised that UCM is not physiologically similar to PBCC and would simulate the haemodynamic instability of immediate UCC.

\section{METHODS}

All experimental procedures were performed in accordance with the National Health and Medical Research Council Code of Practice for the Care and Use of Animals for Scientific Purposes.

\section{Surgical preparation and instrumentation}

Pregnant ewes underwent caesarean sections at $126 \pm 1$ days' gestation (approximately equivalent to 26 weeks' gestational age in humans) under general anaesthesia as previously described. ${ }^{28}{ }^{29}$ Anaesthesia was induced using an intravenous bolus of $5 \%$ sodium thiopentone (pentothal; $1 \mathrm{~g}$ in $20 \mathrm{~mL}$ ) and, following intubation, maintained with inhaled isoflurane $(1.5 \%-3 \%)$ in oxygen/air. Preterm fetal lambs were exposed by hysterotomy and polyvinyl catheters (20 gauge) inserted into the left fetal carotid artery and jugular vein. Flow probes (Transonic Systems, Ithaca, New York, USA) were placed around the right carotid artery, left main pulmonary artery, one umbilical artery and one umbilical vein (sheep have two umbilical veins and arteries). The fetal trachea was intubated with a $4 \mathrm{~mm}$ cuffed endotracheal tube and lung liquid was passively drained prior to ventilation. A near-infrared spectroscopy sensor (Casmed FORE-SIGHT, CAS Medical Systems, Branford, Connecticut, USA) was placed over the left frontal cortex to continuously measure cerebral oxygen saturation $\left(\mathrm{SctO}_{2} \%\right)$.

\section{Experimental intervention}

Twenty-nine preterm lambs were randomised to one of four groups: UCMwoPR $(n=6), \operatorname{UCMwPR}(n=10), \operatorname{PBCC}(n=7)$ and immediate UCC $(n=6)$. UCM involved milking a $10 \mathrm{~cm}$ segment of UC eight times, taking 1-2 s per milk, starting at the placental end and milking towards the fetus. The UC was milked eight times because the UC is shorter in sheep than in humans, allowing only $10 \mathrm{~cm}$ of cord to be milked. In humans, $20 \mathrm{~cm}$ of the UC is commonly milked, and as we aimed to achieve a similar volume of blood transfer in lambs as in human studies, eight milks were required. In lambs receiving UCMwoPR, the UC was released in between milks so that the UC could potentially refill with blood from either the placental or fetal end; this is the technique commonly reported in humans. In lambs receiving UCMwPR, the UC remained occluded after each milk at the fetal end so that blood refilled the UC only from the placental end. The UC was then occluded on the placental end to ensure the UC retained its volume before the milking procedure was repeated (online supplementary videos 1 and 2). In both UCM groups, the UC was clamped immediately after the final milk, without a final release period, and ventilation commenced $30 \mathrm{~s}$ later. In the immediate UCC group, ventilation was initiated $30 \mathrm{~s}$ after the UC was clamped. In the PBCC group, ventilation commenced $3 \mathrm{~min}$ prior to UCC.

\section{Ventilation and monitoring}

In all subjects, ventilation began with a sustained inflation (30 $\mathrm{cmH}_{2} \mathrm{O} \times 30 \mathrm{~s}$ ), followed by volume-guaranteed mechanical ventilation at $7 \mathrm{~mL} / \mathrm{kg}$ with a positive end-expiratory pressure of $5 \mathrm{cmH}_{2} \mathrm{O}$, rate of 60 inflations per minute, inspiratory time of $0.5 \mathrm{~s}$ and fraction of inspired oxygen $\left(\mathrm{FiO}_{2}\right)$ of 0.21 (Dräeger Babylog 8000 Plus ventilator, Dräeger, Lübeck, Germany). The peak inflation pressure was limited to a maximum of $40 \mathrm{cmH}_{2} \mathrm{O}$. Blood gases were recorded immediately prior to commencing the experiment and then at $10 \mathrm{~min}$ intervals (ABL30, Radiometer, Copenhagen, Denmark). Ventilator and $\mathrm{FiO}_{2}$ adjustments were made as needed to maintain arterial $\mathrm{pH}>7.25$, partial pressure of carbon dioxide in arterial blood $\left(\mathrm{PaCO}_{2}\right) 45-55$, and peripheral capillary oxygen saturation $\left(\mathrm{SpO}_{2}\right)$ according to published reference ranges for the first $10 \mathrm{~min}$, then $85 \%-95 \%$ thereafter. ${ }^{30}$ Vital signs and physiological parameters were monitored and recorded continuously using LabChart (ADInstruments, Bella Vista, New South Wales, Australia).

After UCC, an infusion of Alfaxan $(5-15 \mathrm{mg} / \mathrm{kg} / \mathrm{hour}$, Jurox, Rutherford, Australia) in 5\% dextrose was commenced to maintain sedation for the duration of the experiment. All lambs were ventilated for $60 \mathrm{~min}$. Ewes were euthanised following UCC and lambs were euthanised at the conclusion of the experiment, both with an overdose of sodium pentobarbitone $(100 \mathrm{mg} / \mathrm{kg}$ intravenously, Jurox).

\section{Analysis and statistics}

Baseline and fetal data were analysed using the Kruskal-Wallis test to compare groups. A two-way mixed analysis of variance (ANOVA) with post-hoc analysis using Games-Howell was performed to compare continuous variables between intervention groups. One-way repeated-measures ANOVA with Bonferroni correction for multiple comparisons was used for parametric data, or a Friedman's test with Bonferroni correction for multiple comparisons was used for non-parametric data to analyse the changes in continuous variables over time within each group. Normal data are presented as means and SEM, while non-normalised data are presented as medians and IQRs.

There are two veins and arteries in the fetal lamb; therefore, only half of the potential umbilical blood flow was measured. The flow of umbilical blood to the lamb was assigned a positive value, whereas blood flow from the lamb to the placenta was given a negative value. We measured blood flow, in $\mathrm{mL} / \mathrm{min}$, during the intervention in each vessel in relation to its baseline. The total volume of blood to the fetal lamb was calculated by subtracting the umbilical artery volume from the umbilical venous volume in $\mathrm{mL} / \mathrm{kg}$. The umbilical flows and net blood volumes transferred to the lambs were calculated during UCM for the UCM groups and compared with the time between onset of ventilation and UC clamping in the PBCC group ( $3 \mathrm{~min}$ for PBCC). In the PBCC lambs, the average flow for the umbilical vein, artery and the difference (UV-UA) was 
Table 1 Fetal characteristics for each group reported in mean \pm SD

\begin{tabular}{lclllllll}
\hline & $\mathbf{n}$ & $\%$ Male & Weight $(\mathrm{kg})$ & $\mathrm{Hb}$ & $\mathrm{SctO}_{2} \%$ & $\mathrm{pH}$ & $\mathrm{PaCO}_{2}$ & $\mathrm{PaO}_{2}$ \\
\hline UCMwoPR & 6 & 50 & $3.1 \pm 0.4$ & $13.5 \pm 1.3$ & $45 \pm 8$ & $7.28 \pm 0.06$ & $52 \pm 2$ & $22 \pm 6$ \\
UCMwPR & 10 & 60 & $3.3 \pm 0.4$ & $13.2 \pm 1$ & $48 \pm 8$ & $7.29 \pm 0.05$ & $55 \pm 6$ & $28 \pm 5$ \\
PBCC & 7 & 43 & $3.2 \pm 0.6$ & $12.2 \pm 0.8$ & $49 \pm 1$ & $7.23 \pm 0.05$ & $56 \pm 5$ & $23 \pm 6$ \\
Immediate UCC & 6 & 50 & $3.2 \pm 0.7$ & $12.2 \pm 0.6$ & $50 \pm 2$ & $7.29 \pm 0.06$ & $51 \pm 2$ & $25 \pm 9$ \\
\hline
\end{tabular}

There were no baseline differences between groups.

$\mathrm{PaO}_{2}$, arterial oxygen tension; $\mathrm{Hb}$, haemoglobin; PBCC, physiological-based cord clamping; $\mathrm{SctO}_{2} \%$, cerebral oxygenation; UCC, immediate cord clamping; UCMwPR, umbilical cord milking with placental refill; UCMwoPR, umbilical cord milking without placental refill.

measured before and after ventilation onset and compared using a Friedman's test.

PBF, MBP and CABF were measured from heartbeat to heartbeat and compared between groups at the time of cord clamping in immediate UCC, during UCM and immediately before cord clamping in PBCC. HRs and $\mathrm{SctO}_{2} \%$ were measured every second and compared during the first 5 min after UCC.

SPSS V.24 was used for all statistical calculations. Statistical significance was accepted as $\mathrm{P}<0.05$.

\section{RESULTS}

\section{Baseline fetal characteristics}

Fetal characteristics and initial blood gas status were not different between groups (table 1). Basal PBF, MBP, CABF, HR and $\mathrm{SctO}_{2} \%$ were similar in all groups before the experiment. Physiological recordings showing the effects of each UC intervention are shown in figure 1.

\section{Placental transfusion and umbilical blood volume during milking}

Net umbilical blood flow into the lamb (UV-UA) was higher in UCMwPR $(8.8 \mathrm{~mL} / \mathrm{kg}$, IQR $8-10)$ than in UCMwoPR $(0 \mathrm{~mL} /$ $\mathrm{kg}, \mathrm{IQR}-2.8$ to $1.7, \mathrm{P}=0.012)$ and $\mathrm{PBCC}(1.1 \mathrm{~mL} / \mathrm{kg}, \mathrm{IQR}$ -1.3 to $4.3, \mathrm{P}=0.049$ ) (figure $2 \mathrm{~A}, \mathrm{~B}$ ) groups. No net placental to lamb blood transfusion was detected in UCMwoPR and PBCC lambs. The median volume of blood transfused to the lamb during each milk was $1.2 \mathrm{~mL} / \mathrm{kg}$ (IQR $0.7-2.2$ ) for the UCMwoPR group and $0.92 \mathrm{~mL} / \mathrm{kg}$ (IQR $0.3-1.2$ ) for the UCMwPR group. Following release of the UC between each milk, the volume of blood leaving the lamb (indicated by negative value) was $-2 \mathrm{~mL} /$ $\mathrm{kg}(\mathrm{IQR}-2.8$ to -1.1$)$ in the UCMwoPR group and $0.1 \mathrm{~mL} / \mathrm{kg}$ $(-0.1$ to 0.3$)$ in the UCMwPR group.

Umbilical venous and arterial blood flows were significantly reduced in PBCC lambs on ventilation onset $(\mathrm{P}<0.005$ for both umbilical venous and arterial flow). As the reductions were
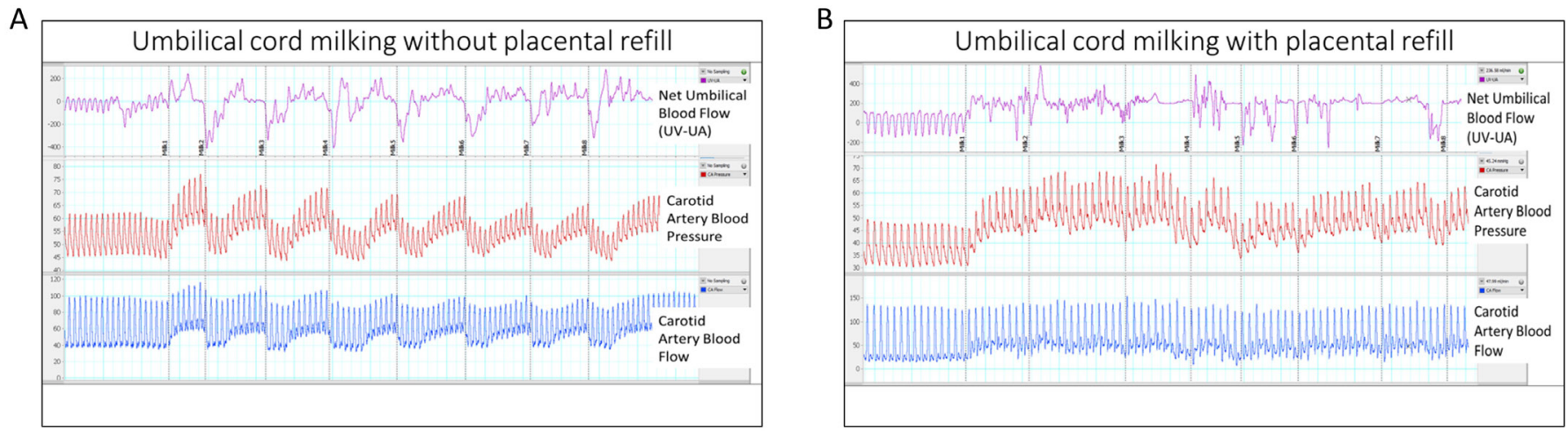

C
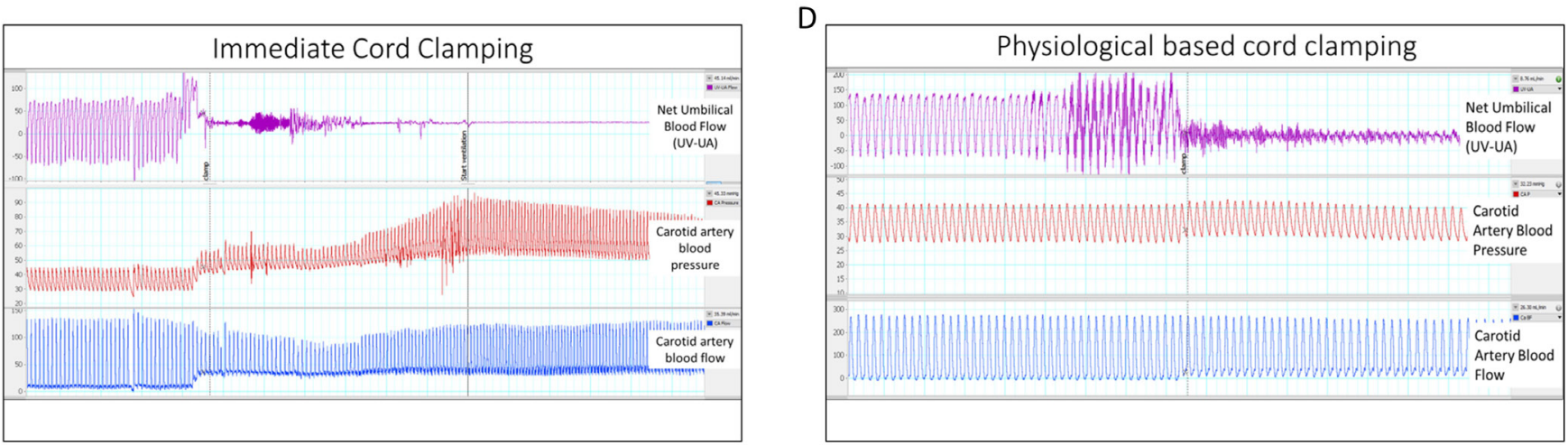

Figure 1 Single animal examples of all four experimental groups. (A) Umbilical cord milking without placenta refill resulted in no net placental transfusion and cerebral haemodynamic instability. (B) Umbilical cord milking with placental refill resulted in a significant increase in placental blood flow to the fetal lamb, but also significant cerebral haemodynamic instability. (C) Immediate cord clamping resulted in significant cerebral haemodynamic instability. (D) Physiological-based cord clamping resulted in haemodynamic stability, but no placental transfusion. UV-UA, umbilical venous flow minus umbilical arterial flow to calculate the net umbilical blood flow toward the fetal lamb. 


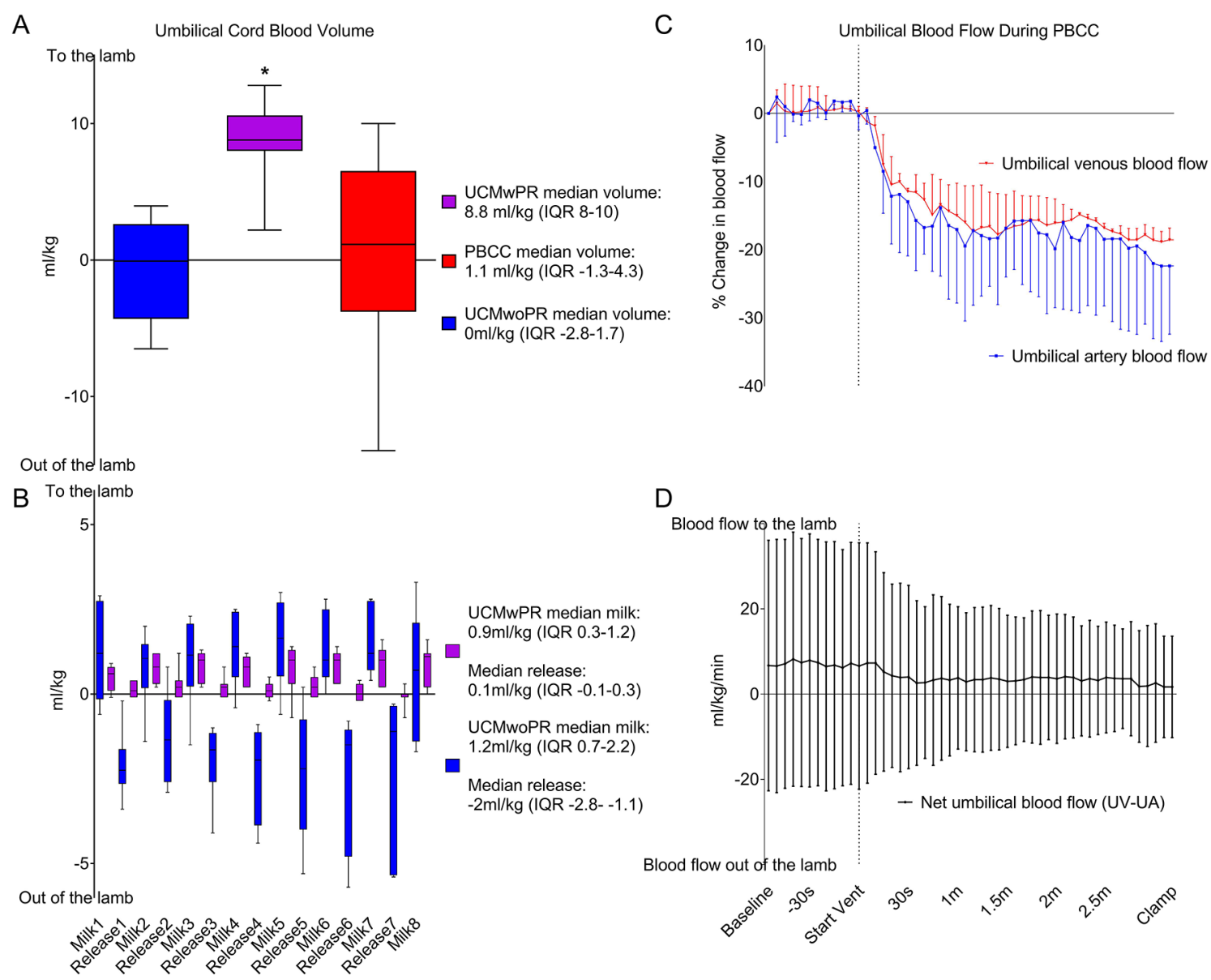

Figure 2 Volume of blood transferred to the lamb during umbilical cord milking and physiological-based cord clamping. (A) Volume of placental transfusion in umbilical cord milking without placental refill (UCMwoPR), umbilical cord milking with placental refill (UCMwPR) and physiologicalbased cord clamping (PBCC). *Indicates significant difference $(\mathrm{P}<0.05)$. (B) Volume and direction of umbilical cord blood volume during UCMwoPR and UCMwPR. (C) Umbilical cord blood flow during the first 3 min of ventilation in PBCC in the umbilical vein and umbilical artery. Umbilical venous and umbilical arterial blood flow were significantly reduced after the initiation of ventilation. (D) Net umbilical blood flow during PBCC (umbilical venous minus umbilical arterial blood flow). Total umbilical blood flow to the fetal lamb did not change during the first 3 min of ventilation.

similar in both vessels, net placental to lamb blood flow did not change during PBCC $(\mathrm{P}=0.999)$ (figure $2 \mathrm{C}, \mathrm{D})$.

\section{Physiological parameters PBF, MBP, CABF, SctO $\%$ and HR}

UCM, either with or without placental refill, was not associated with a change in PBF (UCMwoPR: $2 \pm 1 \mathrm{~mL} / \mathrm{kg} / \mathrm{min}, \mathrm{P}=0.21$; UCMwPR: $1 \pm 1 \mathrm{~mL} / \mathrm{kg} / \mathrm{min}, \mathrm{P}=0.82)$. While immediate UCC also did not increase PBF $(3 \pm 2 \mathrm{~mL} / \mathrm{kg} / \mathrm{min}, \mathrm{P}=0.37)$, PBF was significantly increased prior to UCC in PBCC lambs $(26 \pm 6 \mathrm{~mL} /$ $\mathrm{kg} / \mathrm{min}, \mathrm{P}<0.0005)$. At UCC PBF was significantly greater in PBCC lambs compared with UCMwoPR $(P=0.012)$, UCMwPR $(\mathrm{P}=0.019)$ and immediate UCC $(\mathrm{P}=0.017)$ lambs. PBF was similar in UCMwoPR, UCMwPR and immediate UCC lambs (figure 3).

With each milk, MBP increased by $12 \% \pm 1 \%$ and $8 \% \pm 1 \%$ in UCMwoPR and UCMwPR lambs, respectively, and decreased by $10 \% \pm 1 \%$ and $5 \% \pm 0.5 \%$, respectively, between milks during UC release. In immediate UCC lambs, MBP increased by $46 \% \pm 11 \%(\mathrm{P}<0.0005)$ over the first 70 heartbeats after UCC, whereas ventilation prior to UCC in PBCC lambs resulted in no change in MBP $(5 \% \pm 6 \%, \mathrm{P}=0.46)$ after UCC. Indeed, MBP increased by $16 \% \pm 3 \%$ after eight milks in UCMwoPR lambs $(\mathrm{P}=0.008)$, by $30 \% \pm 3 \%$ in UCMwPR lambs $(\mathrm{P}=0.007)$ and by $46 \% \pm 11 \%$ immediate UCC lambs $(\mathrm{P}<0.0005)$ over the first 70 heartbeats after UCM or UC clamping. Compared with $\mathrm{PBCC}, \mathrm{MBP}$ was increased significantly in response to UCMwoPR $(\mathrm{P}=0.042)$, UCMwPR $(\mathrm{P}=0.021)$ and immediate $\operatorname{UCC}(\mathrm{P}=0.049)$. There were no differences in MBP between UCMwoPR, UCMwPR and immediate UCC (figure 4).

With each milk, CABF increased by $32 \% \pm 2 \%$ and $15 \% \pm 2 \%$ in UCMwoPR and UCMwPR lambs, respectively, and decreased by $23 \% \pm 1 \%$ and $8 \% \pm 1 \%$, respectively, between milks during UC release. During UCM, CABF increased by $40 \% \pm 8 \%(\mathrm{P}<0.0005)$ and $40 \% \pm 2 \%(\mathrm{P}=0.004)$ in UCMwoPR and UCMwPR, respectively, and by $71 \% \pm 11 \%(\mathrm{P}<0.0005)$ in response to immediate UCC. In PBCC lambs no significant change in CABF was observed $(27 \% \pm 10 \%, P=0.1)$ following UCC (figure 4).

$\mathrm{SctO}_{2} \%$ decreased significantly after UCC in all groups, although the decrease was least in PBCC lambs $(-17 \%$, IQR 13-26) compared with UCMwoPR (-26\%, IQR 23-25, $\mathrm{P}=0.03)$, UCMwPR $(-35 \%$, IQR $27-44, \mathrm{P}=0.02)$ and immediate UCC (-34\%, IQR 28-41, P=0.02) lambs (figure 3).

HR did not differ between groups in the 5 min after UCC.

\section{DISCUSSION}

Although UCM is becoming increasingly popular at the delivery of preterm infants, little is known about the acute physiological effects of UCM and how it influences the cardiovascular 
A

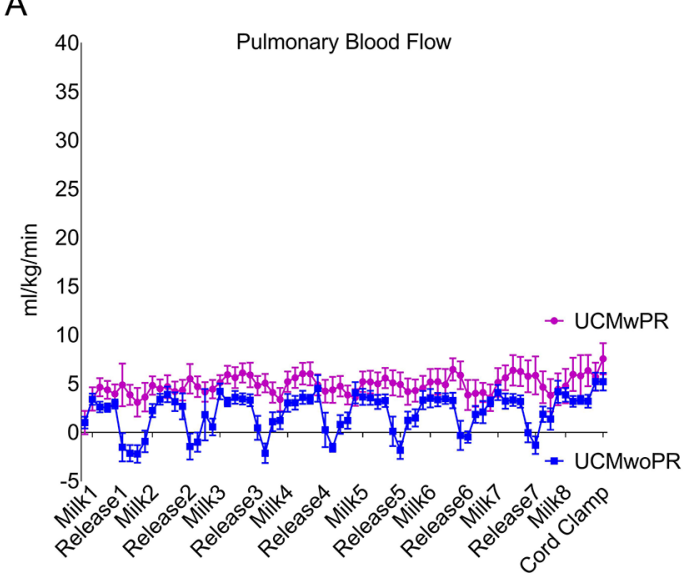

B

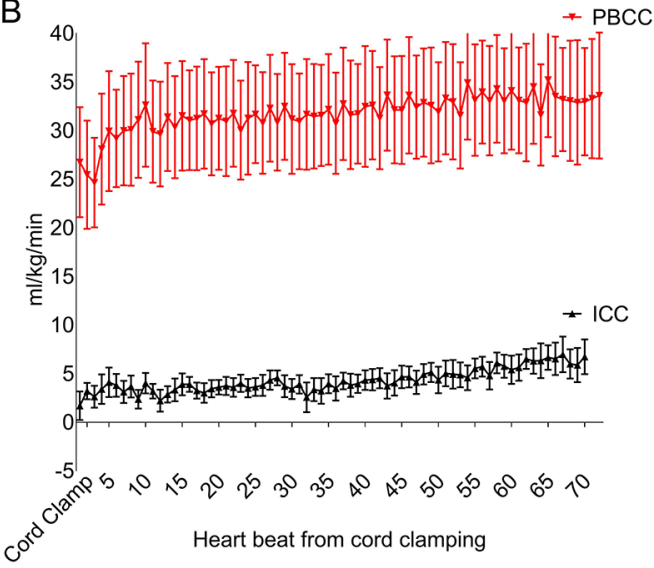

C

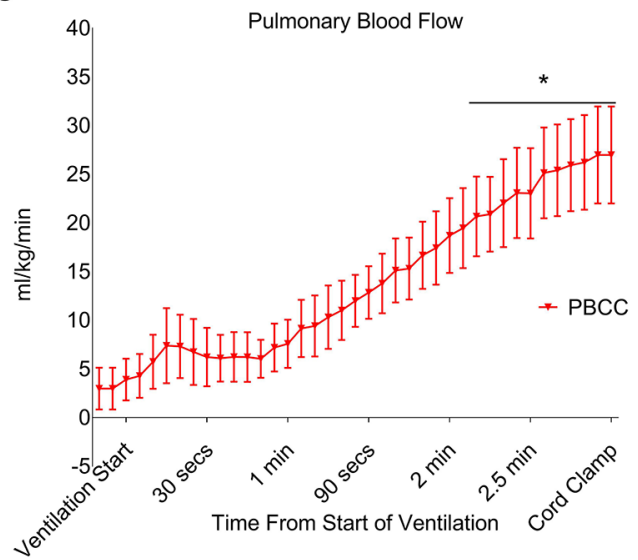

$\mathrm{D}$

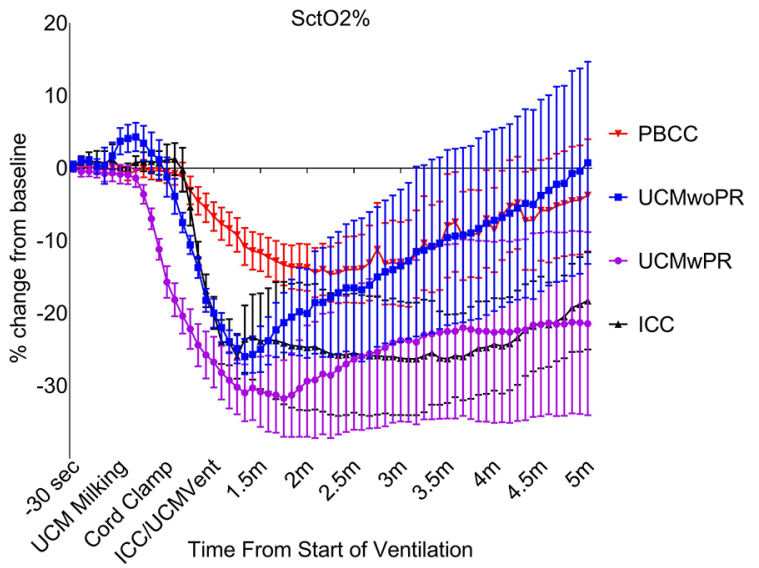

Figure 3 Pulmonary blood flow (PBF) and cerebral oxygenation (SctO $\%$ ). (A) PBF comparing umbilical cord milking without placenta refill (UCMwoPR) and umbilical cord milking with placental refill (UCMwPR) during umbilical cord milking (UCM). (B) PBF comparing physiologicalbased cord clamping (PBCC) and immediate cord clamping (ICC) immediately after cord clamping. (C) PBF in PBCC increased after ventilation. Prior to umbilical cord clamping (UCC), PBF increased by $26 \mathrm{~mL} / \mathrm{kg} / \mathrm{min} \pm 6$ (P $<0.0005)$. (D) SctO $\%$ decreased significantly in all groups after UCC. The decrease was least in PBCC compared with UCMwoPR, UCMwPR and ICC.

transition after birth. We have now quantified the blood transfer achieved by two different UCM strategies and compared the physiological changes associated with UCM with immediate UCC and PBCC strategies. We found that both UCM strategies caused large fluctuations in mean arterial blood pressures and cerebral blood flows that are similar to clamping the UC multiple times. While it is commonly assumed that UCM enhances blood transfer to the fetus, we found that only UCMwPR produced a net blood volume transfer towards the fetus. The measured transfer of approximately $8.8 \mathrm{~mL} / \mathrm{kg}$ (IQR $8-10 \mathrm{~mL} / \mathrm{kg}$ ) of blood during UCMwPR likely represents half the volume the lamb received, as we only measured blood flow in one umbilical vein and one umbilical artery. The volume transfused via UCMwPR is therefore consistent with the reported blood volume transferred during UCM in humans. ${ }^{16} 31$ UCMwoPR caused large fluctuations in blood pressure and cerebral blood flow without any benefit of blood volume transfer. While PBCC greatly mitigated the large oscillations in blood pressure and cerebral blood flow, it did not result in net blood transfer to the lamb. Thus, neither UCM technique was found to be equivalent to PBCC in terms of physiological stability. Both UCM techniques caused major adverse changes in arterial blood pressure and cerebral blood flows and did not stimulate an increase in PBF. As such, neither UCM strategies would be expected to duplicate the benefit of
PBCC and prevent the large decrease in cardiac output that can occur with immediate UCC.

Several trials have examined UCC followed by a single UC milk. ${ }^{16} 181932$ We did not include a group representing this strategy because the UC is significantly shorter in sheep than humans, which would prevent a significant transfusion with this method, and the physiological consequences of immediate UCC in lambs are well known. ${ }^{28} 29$

UCMwoPR did not achieve a net blood transfer to the lamb because blood rapidly refilled the umbilical vessels from the lamb between milks. It has been shown that multiple UCMs prior to UCC (intact-UCM) provide no benefit compared with one time UCM of a $>20 \mathrm{~cm}$ length of cord after UCC (cutUCM) ${ }^{16}$ The most likely explanation for these observations is that during intact UCM, unless blood is only allowed to refill from the placental end, there is only a net transfer of blood to the fetus with the final milk. Nevertheless, the process of milking generated marked haemodynamic fluctuations, with no increase in PBF. We consider this finding to be of concern, as large swings in arterial pressure and cerebral flow caused by UCM are potentially injurious and increase the risk of cerebral vascular injury in preterm infants with an immature cerebral circulation. Similarly, holding the cord above the infant (by $20-30 \mathrm{~cm}$ ) and milking blood down into the infant must generate pressures in 


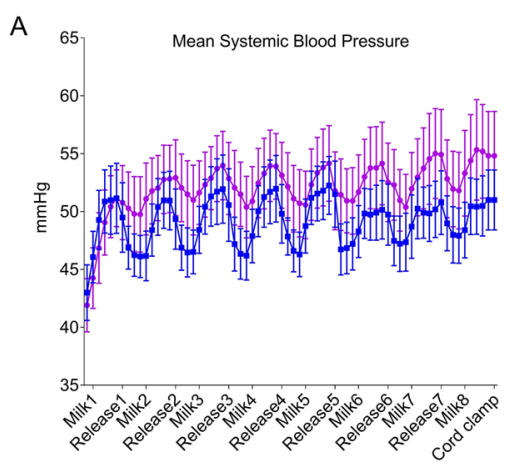

B

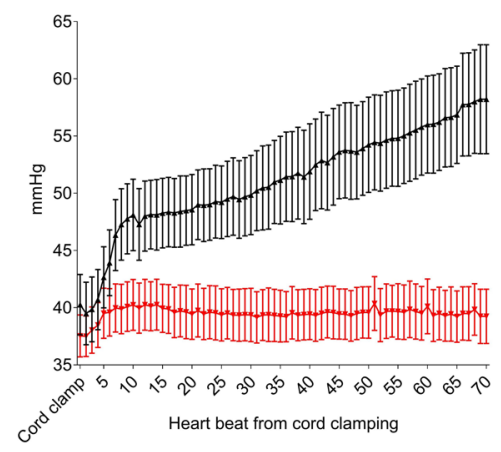

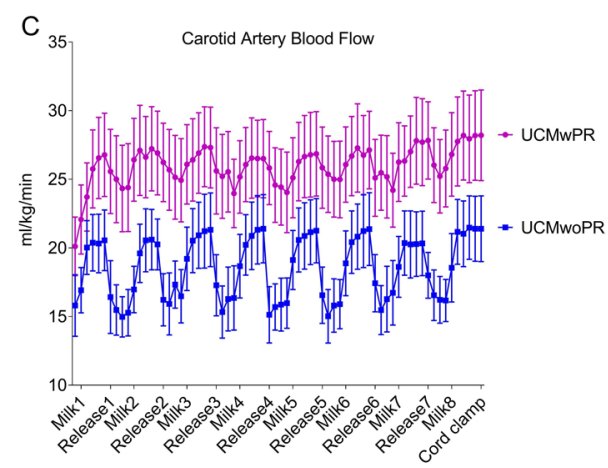

D

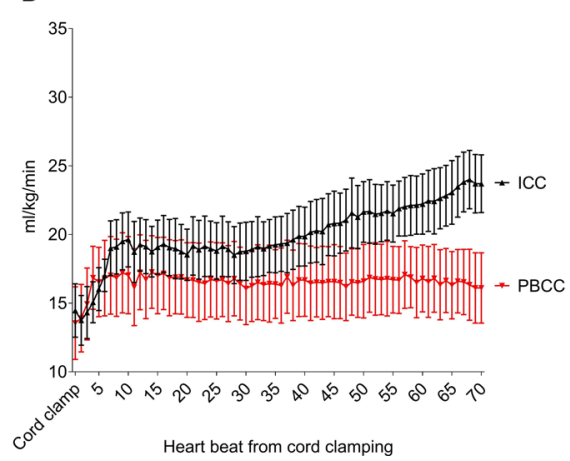

Figure 4 Mean blood pressure (MBP) and mean carotid artery blood flow (CABF) measured heartbeat to heartbeat. (A) MBP comparing umbilical cord milking without placenta refill (UCMwoPR) and umbilical cord milking with placental refill (UCMwPR) during umbilical cord milking. (B) MBP comparing physiological-based cord clamping (PBCC) and immediate cord clamping (ICC) immediately after umbilical cord clamping. (C) CABF comparing UCMwoPR and UCMwPR. (D) CABF comparing PBCC and ICC.

the umbilical vein that are in excess of $20-30 \mathrm{cmH}_{2} \mathrm{O}$, which must dissipate into the central venous/liver portal systems. This is potentially hazardous for the infant and requires further investigation.

Previous animal studies have shown that PBCC, which involves initiating ventilation prior to UCC, greatly mitigates the decrease in cardiac output and the large swings in arterial blood pressure and cerebral blood flow associated with immediate UCC. ${ }^{28} 29$ The same large increases in arterial blood pressure and cerebral blood flow associated with immediate UCC were also observed in UCM (figures 1, 5 and 6), and occurred repeatedly with each milk. As we were able to exactly duplicate these arterial flow and pressure changes by simply occluding and releasing the UC without milking it (data not shown), these changes may simply result from the physiological response to umbilical artery occlusion. While immediate UCC results in a single step-like increase in arterial pressure and flows, UCM results in large oscillating swings in arterial pressures and flows with each milk. As such, depending on the number of milks, UCM has the potential to be considerably more injurious than immediate UCC.

In contrast to UCM and immediate UCC, PBCC produced minimal fluctuations in cerebral blood flow and arterial blood pressure, which is consistent with our previous findings. ${ }^{28}{ }^{29} \mathrm{In}$ addition, as ventilation had commenced prior to UCC, PBCC lambs also had much higher PBFs prior to UCC than all other groups. While it is often assumed that UCM increases PBF, our results clearly demonstrate that UCM does not increase PBF. Instead, PBF only increased following ventilation onset, irrespective of the timing of UCC, once again demonstrating the close relationship between lung aeration and the increase in PBF. In UCM, UCC prior to an increase in PBF reduces cardiac output with the same consequences of immediate UCC lambs. This reduction in cardiac output is caused by a loss of left ventricular preload (no umbilical venous return) combined with an increase in afterload caused by UCC and persists until the lungs aerate and PBF increases. In contrast, PBCC lambs had already increased their PBF prior to UCC, maintaining preload through pulmonary venous return.

We were unable to detect any placental transfusion using the PBCC strategy, which is consistent with our previous findings. ${ }^{33}$ Recent studies in twins (both monochorionic and dichorionic) have shown that when born vaginally, first-born twins have significantly lower haemoglobin levels (at 2 hours and 48 hours after birth) than their second-born twin. ${ }^{3435}$ No differences were observed between first-born and second-born twins delivered by caesarean section, providing compelling evidence that placental transfusion may not occur in infants born by caesarean section. As all our lambs were delivered by caesarean section, this likely explains why we were unable to detect placental transfusion during PBCC. Furthermore, our inability to detect placental transfusion, despite a 10-fold increase in PBF in PBCC lambs, demonstrates that an increase in pulmonary blood volume associated with an increase in PBF is not responsible for placental transfusion. This is consistent with the previous finding that the increase in pulmonary blood volume, associated with pulmonary vascular dilation, is small and can only account for a total increase in blood volume of $\sim 2 \% .{ }^{36}$ Mathematically, this can be explained by the fact that the volume of a tube increases with the increasing radius squared, whereas flow increases with increasing radius to the fourth power.

The lambs in our study had significant respiratory distress and required aggressive ventilation because they were not exposed to antenatal steroids, did not receive surfactant and did not breathe spontaneously. In newborns with exposure to antenatal steroids 
and who begin to breathe spontaneously before UCC, we would expect a larger increase in PBF and more benefit to the PBCC strategy. However, the most appropriate physiological targets for a PBCC-based strategy are unknown.

Our findings are not congruent with the reported clinical studies in that we have failed to demonstrate a significant 'placental transfusion' employing the widely described UCM technique. Repeated clinical studies have inferred a placental transfusion with either delayed UCC or UCM, which is commonly assessed indirectly by measuring haemoglobin levels or weight changes, and attributed improved clinical outcomes to this transfusion ${ }^{1} 10131437$ In our newborn lamb studies, we have repeatedly demonstrated significant haemodynamic fluctuations with cord clamping that are negated if the lamb is ventilated prior to UCC (PBCC). This series of experiments further demonstrates that a 'placental transfusion' from an efficient cord milking technique further compounds the degree of haemodynamic disturbance during transition, suggesting that prior ventilation and not transfusion might be the main advantage of any delay in UCC.

Although this animal model offers considerable advantages in clearly eliciting the physiological changes that are associated with interventions during transition, it has some limitations. All our lambs are delivered by caesarean section in which both the ewe and fetus/lamb are anaesthetised. The majority of premature infants will initiate breathing in the delivery room, which may positively affect PBF and may affect umbilical blood flow. However, our analysis focuses on physiological changes that would occur prior to the initiation of breathing in the majority of preterm infants. ${ }^{11} 1338$ Another limitation of this study is that the fetal lamb undergoes instrumentation prior to the start of the experiment. All groups had similar baseline measurements and normal blood gas values that demonstrated stability. The UC is considerably shorter in sheep than humans and is not as accessible for milking. Lambs have a pair of umbilical veins; thus, we are assuming that blood flows in the measured and unmeasured vessels are similar. Lastly, these animal experiments are conducted only over the transition period and do not provide any information on longer term outcomes, as seen in clinical trials.

\section{CONCLUSIONS}

We have performed the first physiological investigation of UCM, using preterm lambs to test two different UCM techniques. We demonstrated that UCM creates large oscillating swings in arterial pressures and cerebral blood flows with each milk and did not increase PBF. UCMwoPR did not result in a placental transfusion, whereas UCMwPR did. During PBCC, PBF increased prior to UCC and greatly mitigated the large increase in arterial pressures and cerebral blood flows associated with UCC, but did not result in a placental transfusion. Further rigorous evaluation of UCM is required prior to routine clinical use.

Funding PGD (app ID\#1059111) is supported by an Australian National Health and Medical Research Council Practitioner and Principal Research Fellowship. PGD and SBH are supported by the Australian National Health and Medical Research Council Program (\#606789). DB receives a scholarship for his PhD from Monash University. The funders had no role in study design, data collection and analysis, decision to publish, or preparation of the manuscript.

Competing interests None declared.

Ethics approval This is an animal study approved by the Monash University Animal Ethics Committee.

Provenance and peer review Not commissioned; externally peer reviewed.

Open access This is an open access article distributed in accordance with the Creative Commons Attribution Non Commercial (CC BY-NC 4.0) license, which permits others to distribute, remix, adapt, build upon this work non-commercially, and license their derivative works on different terms, provided the original work is properly cited and the use is non-commercial. See: http://creativecommons.org/ licenses/by-nc/4.0/

(c) Article author(s) (or their employer(s) unless otherwise stated in the text of the article) 2018. All rights reserved. No commercial use is permitted unless otherwise expressly granted.

\section{REFERENCES}

1 Rabe H, Diaz-Rossello JL, Duley L, et al. Effect of timing of umbilical cord clamping and other strategies to influence placental transfusion at preterm birth on maternal and infant outcomes. Cochrane Database Syst Rev 2012;8:CD003248.

2 Ersdal HL, Linde J, Mduma E, et al. Neonatal outcome following cord clamping after onset of spontaneous respiration. Pediatrics 2014;134:265-72.

3 Mercer JS, Erickson-Owens DA, Vohr BR, et al. Effects of placental transfusion on neonatal and 18 month outcomes in preterm infants: A randomized controlled trial. J Pediatr 2016;168:50-5.

4 Sommers R, Stonestreet BS, Oh W, et al. Hemodynamic effects of delayed cord clamping in premature infants. Pediatrics 2012;129:e667-e672.

5 Kattwinkel J, Perlman J. The Neonatal Resuscitation Program: The Evidence Evaluation Process and Anticipating Edition 6. NeoReviews 2010;11:e673-e680.

6 Perlman JM, Wyllie J, Kattwinkel J, et al. Neonatal resuscitation: 2010 International Consensus on Cardiopulmonary Resuscitation and Emergency Cardiovascular Care Science with Treatment Recommendations. Pediatrics 2010;126:e1319-e1344.

7 Singhal N, Lockyer J, Fidler H, et al. Helping babies breathe: global neonatal resuscitation program development and formative educational evaluation. Resuscitation 2012;83:90-6.

8 Finer NN, Carlo WA, Walsh MC, et al. Early CPAP versus surfactant in extremely preterm infants. N Engl J Med 2010;362:1970-9.

9 Popat H, Robledo KP, Sebastian L, et al. Effect of Delayed Cord Clamping on Systemic Blood Flow: A Randomized Controlled Trial. J Pediatr 2016;178:81-6.

10 March MI, Hacker MR, Parson AW, et al. The effects of umbilical cord milking in extremely preterm infants: a randomized controlled trial. J Perinatol 2013;33:763-7.

11 Katheria A, Blank D, Rich W, et al. Umbilical cord milking improves transition in premature infants at birth. PLoS One 2014;9:e94085.

12 Katheria AC, Leone TA, Woelkers D, et al. The effects of umbilical cord milking on hemodynamics and neonatal outcomes in premature neonates. J Pediatr 2014; 164:1045-50.

13 Katheria AC, Truong G, Cousins L, et al. Umbilical Cord Milking Versus Delayed Cord Clamping in Preterm Infants. Pediatrics 2015;136:61-9.

14 Rabe H, Jewison A, Alvarez RF, et al. Milking compared with delayed cord clamping to increase placental transfusion in preterm neonates: a randomized controlled trial. Obstet Gynecol 2011;117(Pt 1):205-11.

15 Rabe H, Sawyer A, Amess P, et al. neurodevelopmental outcomes at 2 and 3.5 years for very preterm babies enrolled in a randomized trial of milking the umbilical cord versus delayed cord clamping. Neonatology 2016;109:113-9.

16 Hosono S, Mugishima H, Takahashi S, et al. One-time umbilical cord milking after cord cutting has same effectiveness as multiple-time umbilical cord milking in infants born at $<29$ weeks of gestation: a retrospective study. J Perinatol 2015;35:590-4.

17 Dang D, Zhang C, Shi S, et al. Umbilical cord milking reduces need for red cell transfusions and improves neonatal adaptation in preterm infants: Meta-analysis. J Obstet Gynaecol Res 2015;41:890-5.

18 Takami T, Suganami Y, Sunohara D, et al. Umbilical cord milking stabilizes cerebra oxygenation and perfusion in infants born before 29 weeks of gestation. $J$ Pediatr 2012;161:742-7.

19 Upadhyay A, Gothwal S, Parihar R, et al. Effect of umbilical cord milking in term and near term infants: randomized control trial. Am J Obstet Gynecol 2013;208:120. e1-120.e6.

20 Patel S, Clark EA, Rodriguez CE, et al. Effect of umbilical cord milking on morbidity and survival in extremely low gestational age neonates. Am J Obstet Gynecol 2014;211:519.e1-519.e7

21 Safarulla A. A review of benefits of cord milking over delayed cord clamping in the preterm infant and future directions of research. J Matern Fetal Neonatal Med 2017:30:2966-73.

22 Hooper SB, Fouras A, Siew ML, et al. Expired CO2 levels indicate degree of lung aeration at birth. PLoS One 2013;8:e70895.

23 Hooper SB, Kitchen MJ, Wallace MJ, et al. Imaging lung aeration and lung liquid clearance at birth. Faseb J 2007;21:3329-37.

24 Schmölzer GM, Hooper SB, Wong C, et al. Exhaled carbon dioxide in healthy term infants immediately after birth. J Pediatr 2015;166:844-9.

25 te Pas AB, Davis PG, Hooper SB, et al. From liquid to air: breathing after birth. J Pediatr 2008;152:607-11.

26 van Vonderen JJ, Roest AA, Siew ML, et al. Measuring physiological changes during the transition to life after birth. Neonatology 2014;105:230-42.

27 Blank D, Rich W, Leone T, et al. Pedi-cap color change precedes a significant increase in heart rate during neonatal resuscitation. Resuscitation 2014;85:1568-72. 
28 Polglase GR, Dawson JA, Kluckow M, et al. Ventilation onset prior to umbilical cord clamping (physiological-based cord clamping) improves systemic and cerebral oxygenation in preterm lambs. PLoS One 2015;10:e0117504.

29 Bhatt S, Alison BJ, Wallace EM, et al. Delaying cord clamping until ventilation onset improves cardiovascular function at birth in preterm lambs. J Physiol 2013;591:2113-26.

30 Dawson JA, Kamlin CO, Vento M, et al. Defining the reference range for oxygen saturation for infants after birth. Pediatrics 2010;125:e1340-e1347.

31 Hosono S, Hine K, Nagano N, et al. Residual blood volume in the umbilical cord of extremely premature infants. Pediatr Int 2015;57:68-71.

32 Hosono $\mathrm{S}$, Mugishima H, Fujita $\mathrm{H}$, et al. Umbilical cord milking reduces the need for red cell transfusions and improves neonatal adaptation in infants born at less than 29 weeks' gestation: a randomised controlled trial. Arch Dis Child Fetal Neonatal Ed 2008;93:F14-F19.

33 Hooper SB, Binder-Heschl C, Polglase GR, et al. The timing of umbilical cord clamping at birth: physiological considerations. Matern Health Neonatol Perinatol 2016;2.
34 Verbeek L, Zhao DP, Middeldorp JM, et al. Haemoglobin discordances in twins: due to differences in timing of cord clamping? Arch Dis Child Fetal Neonatal Ed 2017; 102:F324-F328.

35 Verbeek L, Zhao DP, Te Pas AB, et al. Hemoglobin differences in uncomplicated monochorionic twins in relation to birth order and mode of delivery. Twin Res Hum Genet 2016:19:241-5.

36 Walker AM, Alcorn DG, Cannata JC, et al. Effect of ventilation on pulmonary blood volume of the fetal lamb. J App/ Physiol 1975;39:969-75.

37 Mercer JS, Vohr BR, McGrath MM, et al. Delayed cord clamping in very preterm infants reduces the incidence of intraventricular hemorrhage and late-onset sepsis: a randomized, controlled trial. Pediatrics 2006;117:1235-42.

38 Katheria A, Poeltler D, Durham J, et al. Neonatal Resuscitation with an Intact Cord: A Randomized Clinical Trial. J Pediatr 2016:178:75-80. 